\title{
THE AUTOMORPHISM GROUP OF A COMPACT GROUP ACTION
}

BY

\author{
W. D. CURTIS
}

\begin{abstract}
This paper contains results on the structure of the group, Diff $_{G}^{r}(M)$, of equivariant $C^{r}$-diffeomorphisms of a free action of the compact Lie group $G$ on $M$. Diff ${ }_{G}^{r}(M)$ is shown to be a locally trivial principal bundle over a submanifold of $\operatorname{Diff}^{r}(X), X$ the orbit manifold. The structural group of this bundle is $E^{r}(G, M)$, the set of equivariant $C^{r}$-diffeomorphisms which induce the identity on $X . E^{r}(G, M)$ is shown to be a submanifold of $\operatorname{Diff}^{r}(M)$ and in fact a Banach Lie group $(r<\infty)$.
\end{abstract}

0 . Introduction. This paper studies the group of equivariant diffeomorphisms of a smooth action of a compact Lie group $G$ on a compact manifold $M$. Specifically most of the paper deals with the case when $G$ acts freely on $M$. In this case there is an orbit manifold $X$, and an equivariant $C^{r}$-diffeomorphism $f$ of $M$ induces a $C^{r}$-diffeomorphism $\bar{f}$ of $X$. This defines a homomorphism $P$ : $\operatorname{Diff}_{G}^{r}(M) \rightarrow \operatorname{Diff}^{r}(X)$ (Diff ${ }_{G}^{r}(M)$ is the group of equivariant diffeomorphisms on $M$ of class $C^{r}, 1 \leqslant r \leqslant \infty$. We obtain some results about the structure of $P$. We show $P$ admits smooth local cross-sections (Theorem 3.5). The kernel of $P$ is the group of $C^{r}$-equivariant diffeomorphisms of $M$ which induce the identity on $X$. This group is the structural group of the locally trivial principal bundle determined by $P$. We show $\operatorname{ker} P$ is a smooth submanifold of $\operatorname{Diff}^{r}(M)$ and, that with respect to the induced differential structure, $\operatorname{ker} P$ is a Banach Lie group (Theorem 4.2). Recall $\operatorname{Diff}^{r}(M)$ is not a Banach Lie group as composition is not $C^{1}(r<\infty)$.

The main technique introduced is the construction in $\S 2$ of $G$-lifts of sprays on $X$. This allows a precise connection between the manifold of maps differential structures on $\operatorname{Diff}_{G}^{r}(M)$ and $\operatorname{Diff}^{r}(X)$.

1. Preliminaries. $M$ will always denote a compact, connected, $C^{\infty}$-manifold, $G$ a compact Lie group. Assume $G$ acts on $M$ (on the left). We denote by $X$ the orbit space and we let $\pi: M \rightarrow X$ be the orbit projection. If $G$ acts freely and differentiably $\left(C^{\infty}\right)$ then $X$ has a natural $C^{\infty}$-structure such that $\pi$

Presented to the Society, January 26, 1973 under the title The automorphism group of a compact Lie group action; received by the editors March 22, 1973.

AMS (MOS) subject classifications (1970). Primary 58D05, 22E65; Secondary 55F10.

Key words and phrases. Diffeomorphism group, equivariant diffeomorphism, Banach Lie group, principal bundle, spray, free action. 
is $C^{\infty}$ and locally trivial (since $G$ is compact).

If $g \in G, w \in M$ we write $g w$ to denote the result of letting $g$ act on $w$. We shall also write $g$ to denote the diffeomorphism $w \rightarrow g w$ (henceforth all manifolds, actions, etc. are assumed $C^{\infty}$ ). An action of $G$ on $M$ induces an action of $G$ on $T M$, the tangent bundle of $M$. If $g \in G$ we write $T g(v)$ for the result of acting on $v \in T M$ by $g$. The resulting diffeomorphism of $T M$ is $T g: T M \rightarrow T M$ and is just the tangent of $g: M \rightarrow M$. Similarly $G$ acts on $T^{2} M=T(T M)$ by "double tangents", $T^{2} g=T(T g): T^{2} M \rightarrow T^{2} M$ being the diffeomorphism corresponding to the group element $g$.

Definition 1.1. Let $G$ act on $M$. An automorphism of class $C^{r}$ is a diffeomorphism $f: M \rightarrow M$ of class $C^{r}$ which is $G$-equivariant. We denote by $\operatorname{Diff}_{G}^{r}(M)$ the group of all $C^{r}$-automorphisms of the action. (Here $1 \leqslant r \leqslant \infty$.)

Let $\operatorname{Diff}^{r}(M)$ be the group of all $C^{r}$-diffeomorphisms of $M$ onto $M$. If $G$ acts on $M$ then $\operatorname{Diff}_{G}^{r}(M)$ is clearly a subgroup of $\operatorname{Diff}^{r}(M)$.

The differential structure on $\operatorname{Diff}^{r}(M)$ : We review the standard manifold of maps construction as applied to make $\operatorname{Diff}^{r}(M)$ into a $C^{\infty}$-manifold. We recall that $\operatorname{Diff}^{r}(M)$ is locally Banach if $1 \leqslant r<\infty$ while being locally Fréchet in the case $r=\infty$. Also in case $r=\infty$ the multiplication and inversion in $\operatorname{Diff}^{r}(M)$ are $C^{\infty}$ so $\operatorname{Diff}^{\infty}(M)$ is a Fréchet Lie group [5].

Let $\xi: T M \rightarrow T^{2} M$ be a $C^{\infty}$-spray. There is an open neighborhood 0 of the 0 -section in $T M$ and an open neighborhood $U$ of the "diagonal" in $M \times$ $M$ and a $C^{\infty}$-diffeomorphism Exp: $O \rightarrow U$ defined by $\operatorname{Exp}(v)=(\tau(v), \exp (v))$ where $\tau: T M \rightarrow M$ is projection and exp is the exponential of the spray $\xi$. We use Exp to construct "natural charts" around each $f \in \operatorname{Diff}^{r}(M)$. We define a neighborhood $N_{f}$ of $f$ by

$$
N_{f}=\left\{h \in \operatorname{Diff}^{r}(M) \mid(f(x), h(x)) \in U \text { for all } x \in M\right\} .
$$

Letting $\Gamma^{r}\left(f^{*} T M\right)$ denote the space of all $C^{r}$-sections of the induced bundle $f^{*} T M$, with the $C^{r}$-topology, we define $\alpha_{f}: N_{f} \rightarrow \Gamma^{r}\left(f^{*} T M\right)$ by $\alpha_{f}(h)(x)=$ $\left(x, \operatorname{Exp}^{-1}(f(x), h(x))\right) . \alpha_{f}$ maps $N_{f}$ bijectively onto an open set $\Gamma^{r}\left(f^{*} T M\right)$ and the collection of all charts $\left(N_{f}, \alpha_{f}\right)$ for $f \in \operatorname{Diff}^{r}(M)$ is a $C^{\infty}$-atlas. This $C^{\infty}$-structure is independent of the choice of spray. For further discussion of this manifold of maps construction, we refer to [1], [3], [5].

For use later we now consider some properties of $G$-invariant sprays.

DEFInItion 1.2. If $G$. acts on $M$ we say a spray $\xi: T M \rightarrow T^{2} M$ is $G$-invariant if, for all $g \in G$, we have $T^{2} g \circ \xi=\xi \circ T g$.

The following proposition is easily proved.

Proposition 1.3. If $\xi$ is a G-invariant spray, then the domain of $\exp _{\xi}$ is an invariant subset of $T M$ and $\exp _{\xi}$ is G-equivariant. 
Using notation as before we have

Lemma 1.4. If $\xi$ is a G-invariant spray then 0 may be chosen to be G-invariant. Exp: $O \rightarrow M \times M$ is then G-equivariant, where $G$ acts on $M \times$ $M$ by $g\left(x_{1}, x_{2}\right)=\left(g x_{1}, g x_{2}\right)$. It then follows that $U=\operatorname{Exp}(O)$ is an invariant set.

Proof. Let $O_{1}$ be a neighborhood of the 0 -section such that Exp is a diffeomorphism on $O_{1}$. If we choose a $G$-invariant Riemannian metric on $M$, then for sufficiently small $\epsilon>0$ we will have $W_{\epsilon}=\{v \in T M \mid\|v\|<\epsilon\} \subset O_{1}$ (since $M$ is compact). But $W_{\epsilon}$ is $G$-invariant since we have used an invariant metric. If $g \in G, v \in O=W_{\epsilon}$ we have $\operatorname{Exp}(T g \cdot v)=(\tau(T g \cdot v), \exp (T g \cdot v))=$ $(g \tau(v), g \exp (v))=g \operatorname{Exp}(v)$. This proves the lemma.

The group Diff ${ }_{G}^{r}(M)$ : If $G$ acts freely on $M$ then there is a homomorphism $P: \operatorname{Diff}_{G}^{r}(M) \rightarrow \operatorname{Diff}^{r}(X)$. The main results of this paper are concerned with the structure of $P . P$ is defined by $P(f)=f_{1}$ where $f_{1}$ makes the following diagram commute.

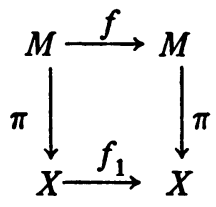

A preliminary result is

Proposition 1.5. Diff $G_{G}^{r}(M)$ is a submanifold of $\operatorname{Diff}^{r}(M)$ for any action of $G$ on $M$ (free or not).

Proof. Let $\xi$ be an invariant spray. Such always exist, e.g. take $\xi$ to be the geodesic spray of an invariant Riemann metric. We show that the natural chart at the identity map is a submanifold chart. We have

$$
\alpha: N \rightarrow \Gamma^{r}(T M), \quad \alpha(f)(x)=\operatorname{Exp}^{-1}(x, f(x)):
$$

Let $\Gamma_{G}^{r}(T M) \subset \Gamma^{r}(T M)$ consist of all $C^{r}$-vectorfields $\zeta$ on $M$ such that $T g \circ \zeta=\zeta \circ g$ for all $g \in G$ (i.e., all invariant vectorfields). Using the fact that by Lemma 1.4 Exp is equivariant, one immediately sees that $\alpha\left(N \cap \operatorname{Diff}_{G}^{r}(M)\right)=$ $\alpha(N) \cap \Gamma_{G}^{r}(T M)$. This proves the proposition.

Assumption. Henceforth we consider only free actions of $G$ on $M$.

In order to investigate the homomorphism $P$ defined above we shall, in the next section, construct a special type of spray on $M$. This spray will allow us to relate the differential structure on $\operatorname{Diff}_{G}^{r}(M)$ to that on $\operatorname{Diff}^{r}(X)$.

2. Construction of $G$-lifts of sprays. Suppose $G$ acts freely on $M, \pi$ : $M \rightarrow X$ orbit projection. If $\eta$ is a spray on $X$ then a spray on $M$, say $\xi$, 
is called a lift of $\eta$ if the following commutes.

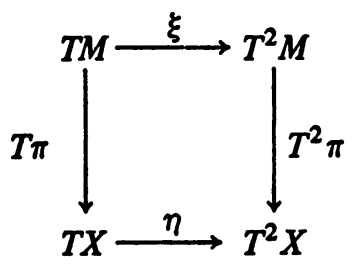

If, in addition, $\xi$ is $G$-invariant we will say $\xi$ is a $G$-lift of $\eta$. We shall prove

THEOREM 2.1. If $\eta$ is any spray on $X$ then there exists a spray $\xi$ on $M$ which is a G-lift of $\eta$.

The proof of Theorem 2.1 will be based on several lemmas.

Let $\left\{U_{i}\right\}_{t}$ ( $i$ in some index set) be an open cover for $X$. Let $M_{i}=$ $\pi^{-1}\left(U_{i}\right)$ for each $i$. $\left\{M_{i}\right\}_{i}$ is an open cover of $M$ and $\left\{T M_{i}=\tau^{-1}\left(M_{i}\right)\right\}_{i}$ is an open cover of $T M$.

LEMMA 2.2. There is a $C^{\infty}$-partition of unity $\left\{\varphi_{i}\right\}_{i}$, subordinate to $\left\{T M_{i}\right\}_{i}$ such that each $\varphi_{i}$ is G-invariant, i.e., $\varphi_{i} \circ T g=\varphi_{i}$ for all $g \in G$.

Proof. Choose a $C^{\infty}$-partition of unity, $\left\{\psi_{i}\right\}_{i}$ subordinate to $\left\{U_{i}\right\}_{i}$. Let $\varphi_{i}=\psi_{i} \circ \pi \circ \pi .\left\{\varphi_{i}\right\}_{i}$ is easily seen to have the desired properties.

The next lemma is general in nature. Let $Y, Z$ be $C^{\infty}$-manifolds, $\xi, \eta$ $C^{\infty}$-sprays on $Y, Z$. There are vector bundle isomorphisms

$\alpha: T(Y \times Z) \rightarrow T Y \times T Z, \quad \alpha(v)=\left(T \pi_{1}(v), T \pi_{2}(v)\right)$,

$\beta: T(T Y \times T Z) \rightarrow\left(T^{2} Y\right) \times\left(T^{2} Z\right), \quad \beta(2)=\left(T \pi_{1}(w), T \pi_{2}(w)\right)$.

Thus we have a bundle isomorphism

$$
\delta=\beta \circ T \alpha: T^{2}(Y \times Z) \rightarrow\left(T^{2} Y\right) \times\left(T^{2} Z\right) .
$$

LEMMA 2.3. Let $\gamma$ be the unique map making the following diagram commute. Then $\gamma$ is a spray on $Y \times Z$. (We shall write $\xi * \eta$ for $\gamma$.)

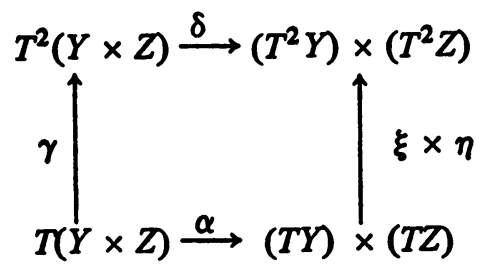

Proof. If $Y$ is modelled on $E$ and $Z$ is modelled on $F$, then the local version of the above diagram is

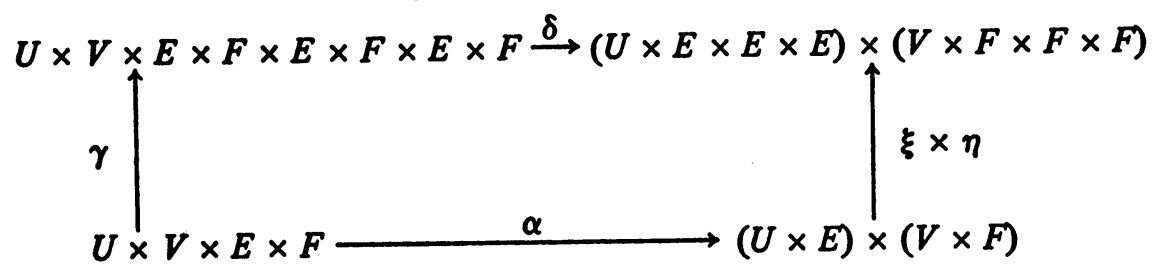


We have:

$$
\begin{aligned}
\alpha(x, y, u, v) & =(x, u, y, v), \\
\delta\left(x, y, u, v, u^{\prime}, v^{\prime}, u^{\prime \prime}, v^{\prime \prime}\right) & =\left(x, u, u^{\prime}, u^{\prime \prime}, y, v, v^{\prime}, v^{\prime \prime}\right) .
\end{aligned}
$$

If $\bar{\xi}, \bar{\eta}$ are the principal parts of $\xi, \eta$ we easily see

$$
\gamma(x, y, u, v)=(x, y, u, v, u, v, \bar{\xi}(x, u), \bar{\eta}(y, v)) .
$$

This local form shows $\gamma$ is a spray.

Lemma 2.4. Let notation be as above, $p: Y \times Z \rightarrow Y$ projection. Then the following diagram commutes.

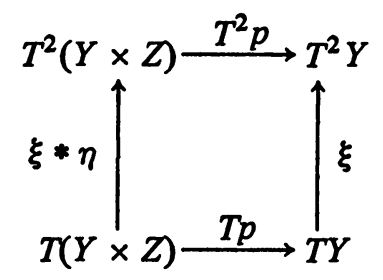

Proof. This is immediate from the local representation of Lemma 2.3, or an invariant proof can be given. We omit details.

Now return to $\pi: M \rightarrow X$. Suppose $U \subset X$ is open and $\varphi: \pi^{-1}(U) \rightarrow$ $U \times G$ is an equivariant diffeomorphism such that $\pi_{1} \circ \varphi=\pi$. ( $G$ acts on $U \times G$ by $g\left(x, g^{\prime}\right)=\left(x, g g^{\prime}\right)$.) Recall that $X$ is covered by such $U$. If $\eta$ is a fixed spray on $X$, then $\eta$ induces a spray on $U$ which we denote $\eta_{U}$. Let $\mu: T G \rightarrow T^{2} G$ be the canonical left-invariant spray of $G[6$, p. 222].

Then $\eta_{U} * \mu$ is a spray on $U \times G$.

Lemma 2.5. Let $G$ act on $U \times G$ as above. Then $\eta_{U} * \mu$ is G-invariant.

Proof. Let $v \in T(U \times G), \alpha(v)=\left(v_{1}, v_{2}\right)$. Now

$$
\begin{aligned}
T^{2} g \circ\left(\eta_{U} * \mu\right)(v) & =T^{2} g \circ(T \alpha)^{-1} \circ \beta^{-1} \circ\left(\eta_{U} \times \mu\right)\left(v_{1}, v_{2}\right) \\
& =T\left(T g \circ \alpha^{-1}\right) \circ \beta^{-1}\left(\eta_{U}\left(v_{1}\right), \mu\left(v_{2}\right)\right) \\
& =T\left(\alpha^{-1} \circ(1 \times T g)\right) \circ \beta^{-1}\left(\eta_{U}\left(v_{1}\right), \mu\left(v_{2}\right)\right) \\
& =T \alpha^{-1} \circ \beta^{-1} \circ\left(1 \times T^{2} g\right)\left(\eta_{U}\left(v_{1}\right), \mu\left(v_{2}\right)\right) \\
& =T \alpha^{-1} \circ \beta^{-1}\left(\eta_{U}\left(v_{1}\right), T^{2} g \circ \mu\left(v_{2}\right)\right) \\
& =T \alpha^{-1} \circ \beta^{-1}\left(\eta_{U}\left(v_{1}\right), \mu\left(T g\left(v_{2}\right)\right)\right) \\
& =T \alpha^{-1} \circ \beta^{-1} \circ\left(\eta_{U} \times \mu\right)\left(v_{1}, T g\left(v_{2}\right)\right) \\
& =\eta_{U} * \mu(T g(v)) .
\end{aligned}
$$


Let $\xi_{U}$ be the spray on $\pi^{-1}(U)$ such that the following diagram commutes.

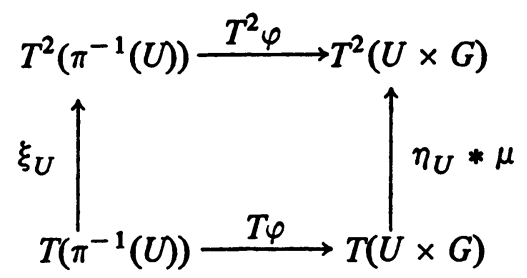

Since $\varphi$ is an equivariant diffeomorphism, we see $\xi_{U}$ is a $G$-invariant spray on $\pi^{-1}(U)$.

We can now prove Theorem 2.1.

Proof of Theorem 2.1. Cover $X$ by open sets $U_{i}$ such that for each $i$ there is an equivariant diffeomorphism $\varphi_{i}: \pi^{-1}\left(U_{i}\right) \rightarrow U_{i} \times G$ as above. Let $\xi_{i}=\xi_{U_{i}}$ be as above. Let $\left\{\varphi_{i}\right\}_{i}$ be an invariant partition of unity subordinate to $\left\{T M_{i}\right\}_{i}$ as in Lemma 2.2. Let $\xi=\Sigma_{i} \varphi_{i} \xi_{i}: T M \rightarrow T^{2} M$. $\xi$ is a spray on $M$. Using the invariance of the $\varphi_{i}$ we easily see $\xi$ is invariant. It remains to show $\xi$ is a lift of $\eta$. Let $v \in T M$.

$$
T^{2} \pi \circ \xi(v)=T^{2} \pi\left(\sum_{i} \varphi_{i}(v) \xi_{i}(v)\right)=\sum_{i} \varphi_{i}(v) T^{2} \pi \circ \xi_{i}(v) .
$$

Suppose $T^{2} \pi \circ \xi_{i}(v)=\eta(T \pi(v))$ for each $i$ with $v \in T M_{i}$. Then we will be done. If $v \in T M_{i}$ we compute as follows:

$$
\begin{aligned}
T^{2} \pi\left(\xi_{i}(v)\right) & =T^{2} \pi\left(T^{2} \varphi_{i}^{-1} \circ\left(\eta_{U} * \mu\right) \circ T \varphi_{i}(v)\right) \\
& =T^{2}\left(\pi \circ \varphi_{i}^{-1}\right) \circ\left(\eta_{U_{i}} * \mu\right) \circ T \varphi_{i}(v)=T_{p}^{2} \circ \eta_{U_{i}} * \mu \circ T \varphi_{i}(v) \\
& =\eta_{U_{i}} \circ T p\left(T \varphi_{i}(v)\right)=\eta_{U_{i}} \circ T \pi(v)=\eta(T \pi(v)) .
\end{aligned}
$$

Here $p: U_{i} \times G \rightarrow U_{i}$ is projection and we have used Lemma 2.4. This completes the proof of Theorem 2.1.

3. The structure of $P$. Henceforth $\eta$ will be a fixed spray on $X$ and $\xi$ will be a fixed $G$-lift of $\eta$. Recall that the domain of $\exp _{\xi}$ is $\operatorname{Dom}\left(\exp _{\xi}\right)=$ $\left\{v \in T M \mid 1 \in \operatorname{Dom}\left(\beta_{v}\right)\right\}$ where $\beta_{v}$ is the maximal integral curve of $\xi$ with initial condition $v$. Similarly $\operatorname{Dom}\left(\exp _{\eta}\right)=\left\{w \in T X \mid 1 \in \operatorname{Dom}\left(\gamma_{w}\right)\right\}$ where $\gamma_{w}$ is the maximal integral curve of $\eta$ with initial condition $w$. Since $\xi$ is a lift of $\pi$ we see $\xi$ and $\eta$ are $T \pi$-related, so $T \pi\left(\operatorname{Dom}\left(\exp _{\xi}\right)\right) \subset \operatorname{Dom}\left(\exp _{\eta}\right)$ and the following diagram commutes.

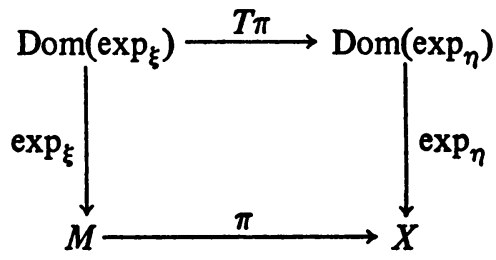


We shall use $\xi$ and $\eta$ to construct the differential structures on $\operatorname{Diff}^{r}(M)$ and $\operatorname{Diff}^{r}(X)$ respectively. Using the above properties of $\xi$ and $\eta$ we easily see we may choose open sets $0, \delta$ containing the 0 -sections of $T M, T X$, respectively, such that the following hold: (1) $O$ is $G$-invariant; (2) $T \pi(0)=\bar{O}$; (3) $\operatorname{Exp}_{\xi}$ and $\operatorname{Exp}_{\eta}$ map 0 and $\delta$ diffeomorphically onto neighborhoods $U$ and $\bar{U}$ of the respective diagonals; (4) $(\pi \times \pi)(U)=\bar{U}$. Also, we clearly have $(\pi \times \pi) \circ \operatorname{Exp}_{\xi}=\operatorname{Exp}_{\eta} \circ T \pi$.

As in $\S 1$ we get natural charts $(N, \alpha),(\bar{N}, \bar{\alpha})$ at the identities in $\operatorname{Diff}^{r}(M)$ and $\operatorname{Diff}^{r}(X)$. Since $\xi$ is an invariant spray we see, as in Proposition 1.5, we get a submanifold chart at $1_{M},\left(N^{*}, \alpha^{*}\right)$ where $N^{*}=N \cap \operatorname{Diff}_{G}^{r}(M), \alpha^{*}=\alpha \mid N^{*}$ : $N^{*} \rightarrow \Gamma_{G}^{r}(T M)$.

Let $\operatorname{Diff}_{0}^{r}(X)$ be the group of all $C^{r}$-diffeomorphisms, $h$, of $X$ such that there is an equivariant $C^{r}$-diffeomorphism of $M$ with $\pi \circ f=h \circ \pi$ (i.e., $f$ covers $h$ ). It is easily seen that $\operatorname{Diff}_{0}^{r}(X)$ is an open subgroup of $\operatorname{Diff}^{r}(X)$ and hence a manifold. Clearly the map $P$ defined earlier is a surjective homomorphism $P: \operatorname{Diff}_{G}^{r}(M) \rightarrow \operatorname{Diff}_{0}^{r}(X)$. Let $\bar{N}^{*}=\bar{N} \cap \operatorname{Diff}_{0}^{r}(X), \bar{\alpha}^{*}=\bar{\alpha} \mid \bar{N}^{*}$, so that $\left(\bar{N}^{*}, \bar{\alpha}^{*}\right)$ is a chart at the identity on $\operatorname{Diff}_{0}^{r}(X)$.

LEMMA 3.1. $P\left(N^{*}\right) \subset \bar{N}^{*}$.

Proof. Let $f \in N^{*}, P(f)=\bar{f}$. We must show $(x, \bar{f}(x)) \in \bar{U}$ for each $x \in X$. Let $x=\pi(y)$. Then $(x, \bar{f}(x))=(\pi \times \pi)(y, f(y))$ as is easily seen. But $(y, f(y)) \in U$ and $(\pi \times \pi)(U)=\bar{U}$ so the lemma follows.

Let $\rho$ be the unique map making the following diagram commute.

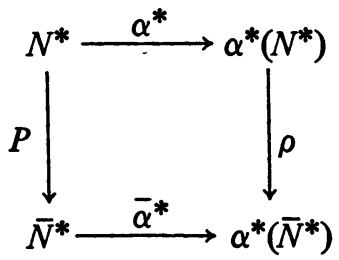

LEMMA 3.2. There is a continuous linear map, $\rho_{1}: \Gamma_{G}^{r}(T M) \rightarrow \Gamma^{r}(T X)$, such that $\rho_{1} \mid \alpha^{*}\left(N^{*}\right)=\rho$.

Proof. We first show that for $\zeta \in \Gamma_{G}^{r}(T M)$ there is a unique map, $\rho_{1}(\zeta)$ in $\Gamma^{r}(T X)$ such that $T \pi \circ \zeta=\rho_{1}(\zeta) \circ \pi$. If $\pi(u)=\pi(v)$ we must show $T \pi(\zeta(u))=T \pi(\zeta(v))$. If $\pi(u)=\pi(v)$ then $v=g u$ for some $g \in G$. But then $T \pi(\zeta(v))=T \pi(\zeta(g u))=T \pi(T g \circ \zeta(u))=T \pi(\zeta(u))$. This defines $\rho_{1}$ and, working locally, one easily verifies that $\rho_{1}$ is continuous linear and, in particular, that $\rho_{1}(\zeta)$ is actually in $\Gamma^{r}(T X)$. Let $h \in N^{*}, v \in M$. Then

$$
\begin{aligned}
\rho_{1}\left(\alpha^{*}(h)\right)(\pi(v)) & =T \pi\left(\alpha^{*}(h)(v)\right)=T \pi\left(\exp _{\xi}^{-1}(v, h(v))\right) \\
& =\operatorname{Exp}_{\eta}^{-1}(\pi(v), P(h)(\pi(v)))=\bar{\alpha}^{*}(P(h))(\pi(v)) .
\end{aligned}
$$

This proves $\rho_{1} \mid \alpha^{*}\left(N^{*}\right)=\rho$. 
It follows from Lemma 3.2 that $P$ is $C^{\infty}$ in a neighborhood of the identity. Since $P$ is a homomorphism and right translation is $C^{\infty}$ it follows $P$ is $C^{\infty}$ everywhere.

Proposition 3.3. $\rho_{1}$ is a continuous linear surjection with split kernel.

Proof. Recall there is a bundle $V T(M)$ of vertical vectors. $V T(M)=$ $\operatorname{ker}(T \pi) \subset T M$. $V T(M)$ is a $G$-invariant subbundle of $T M$. Let $H$ be an invariant subbundle of $T M$ which is complementary to $V T(M)$ ( $\pi$ is essentially a principal $G$-bundle so $H$ is just an affine connection. See [4].) Let $\Gamma_{G}^{r}(V T(M))$ and $\Gamma_{G}^{r}(H)$ be the spaces of $G$-invariant $C^{r}$-sections of the two subbundles. We immediately have $\operatorname{ker} \rho_{1}=\Gamma_{G}^{r}(V T(M))$. Using the $G$-invariance of the subbundle it is easy to see $\Gamma_{G}^{r}(T M)=\Gamma_{G}^{r}(V T(M)) \oplus \Gamma_{G}^{r}(H)$. So $\Gamma_{G}^{r}(H)$ is a complement for $\operatorname{ker}\left(\rho_{1}\right)$.

That $\rho_{1}$ is onto is just the well-known existence of the horizontal lift of a vectorfield with respect to an affine connection on a principal bundle. (See [4].)

COROLlary 3.4. $\quad \operatorname{ker} P$ is a split submanifold of $\operatorname{Diff}_{G}^{r}(M)$.

Proof. $\alpha^{*}\left(N^{*} \cap \operatorname{ker} P\right)=\alpha^{*}\left(N^{*}\right) \cap \Gamma_{G}^{r}(V T(M))$, as is easily checked. The corollary follows.

THEOREM 3.5. $P$ admits smooth local cross-sections.

Proof. Let $\lambda_{1}: \Gamma^{r}(T X) \rightarrow \Gamma_{G}^{r}(T M)$ be continuous linear with $\rho_{1} \circ \lambda_{1}=$ identity. Choose an open set $W_{1}$ containing 0 in $\Gamma^{r}(T X)$ such that $\lambda_{1}\left(W_{1}\right) \subset$ $\alpha^{*}\left(N^{*}\right)$. Let $W=\left(\bar{\alpha}^{*}\right)^{-1}\left(W_{1}\right)$ and $\lambda(h)=\left(\alpha^{*}\right)^{-1} \circ \lambda_{1} \circ \bar{\alpha}^{*}(h)$ for $h \in W$. Then $h$ is a smooth section of $P$ near the identity so the theorem is proved.

Let $E^{r}(G, M)=\operatorname{ker} P . E^{r}(G, M)$ is the group of self-equivalences of the bundle $\pi$ of class $C^{r}$. In case $r=\infty$ we see $E^{r}(G, M)$ is a Fréchet Lie group since it is a subgroup and closed submanifold of $\operatorname{Diff}^{\infty}(M)$ which is a Fréchet Lie group [5].

If $r<\infty$ we cannot argue the same way since $\operatorname{Diff}^{r}(M)$ is not a Banach Lie group. In the next section we show that, nonetheless, $E^{r}(G, M)$ is a Banach Lie group. The following is immediate from Theorem 3.5 and Corollary 3.4.

THEOREM 3.6. $P: \operatorname{Diff}_{G}^{r}(M) \rightarrow \operatorname{Diff}^{r}(X)$ is a principal bundle with group $E^{r}(G, M)$. If $r=\infty$ this bundle is $C^{\infty}$ while if $r<\infty$ we have a continuous bundle.

The following problem is unsolved.

Determine conditions on $\pi$ so that the bundle $P$ is trivial, i.e., so that $P$ admits a global cross-section. (If $\pi$ is trivial, then so is $P$, as is easily seen.)

4. The group $E^{r}(G, M)$. In this section we assume $r<\infty$. We know by Corollary 3.4 that $E^{r}(G, M)$ is a $C^{\infty}$-Banach manifold. We shall prove that 
$E^{r}(G, M)$ is a Banach Lie group which is $C^{\infty}$-anti-isomorphic to a Banach Lie subgroup of $C^{r}(M, G)$.

In [2] it was shown that $C^{r}(M, G)$ is a Banach Lie group. There is a Banach Lie subgroup $E$ of $C^{r}(M, G)$ defined by

$$
E=\left\{h \in C^{r}(M, G) \mid h\left(g^{\prime} v\right)=g h(v) g^{-1}, \text { for all } g \in G, v \in M\right\} .
$$

$E$ acts on the right of $M$ by $v * h=h(v) v$. (These things are discussed in [2] in more detail, the only difference being that in [2] $G$ acts on the right and $E$ on the left.) This action defines an injective antihomomorphism $\Phi: E \rightarrow \operatorname{Diff}_{G}^{r}(M)$ and $\Phi(E)=E^{r}(G, M)$ as one easily sees.

THEOREM 4.1. $\Phi$ maps diffeomorphically onto $E^{r}(G, M)$.

Proof. It is enough to show that $\Phi$ and $\Phi^{-1}$ are $C^{\infty}$ in some neighborhoods of the respective identities. There is a map $\Phi^{*}: C^{r}(M, G) \rightarrow C^{r}(M, M)$ defined by $\Phi^{*}(f)(v)=f(v) v=\mu(f(v), v)$ where $\mu: G \times M \rightarrow M$ is the group action. This is a $C^{\infty}$-map because there is a commutative diagram

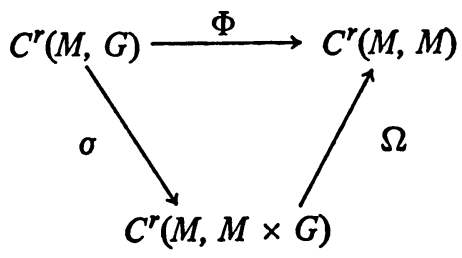

where $\sigma(f)=\left(1_{M}, f\right), \Omega(k)=\mu \circ k$. Both $\sigma$ and $\Omega$ are easily seen to be $C^{\infty}$. $\Phi=\Phi^{*} \mid E$ so $\Phi$ is $C^{\infty}$.

Now consider $\Phi^{-1}$. Recall we have $O \subset T M . \Gamma^{r}(V T(M) \cap 0)$ is the set of $C^{r}$-vectorfields on $M$ which take values in $V T(M) \cap 0$. We define $\psi$ : $\Gamma^{r}(V T(M) \cap 0) \rightarrow C^{r}(M, G)$. Let $M \times_{X} M$ be the submanifold of $M \times M$ consisting of all $(v, w)$ such that $\pi(v)=\pi(w)$. There is a $C^{\infty}$-map $\theta: M \times{ }_{X}$ $M \rightarrow G$ by $\theta(v, w)=g$ if $w=g v$. Since $G$ acts freely, this is well defined and $\theta$ is $C^{\infty}$ as is seen by working locally. Now $\exp _{\xi}$ maps $\operatorname{VT}(M) \cap 0$ into $M \times_{X} M$ as the following calculation shows. Let $v \in V T(M) \cap 0$. Then

$$
\begin{aligned}
(\pi \times \pi)\left(\operatorname{Exp}_{\xi}(v)\right) & =\operatorname{Exp}_{\eta}(T \pi(v))=\left(\tau(T \pi(v)), \exp _{\eta}(T \pi(v))\right) \\
& =\left(\pi(\tau(v)), \exp _{\eta}(0)\right)=(\pi(\tau(v)), \pi(\tau(v)))
\end{aligned}
$$

where 0 is the zero vector in the tangent space to $X$ at $\pi(\tau(v))$.

Thus we have a $C^{\infty}$-map $\psi: \Gamma^{r}(V T(M) \cap 0) \rightarrow C^{r}(M, G)$ by $\psi(\xi)=$ $\theta \circ \operatorname{Exp}_{\xi} \circ \xi$. We show that $\psi$ takes the subspace $\Gamma_{G}^{r}(V T(M) \cap 0)$ into the subgroup $E$ of $C^{r}(M, G) . G$ acts on $M \times_{X} M$ by $g(v, w)=(g v, g w)$ and one easily sees $\theta(g v, g w)=g \theta(v, w) g^{-1}$. Thus if $\zeta \in \Gamma_{G}^{r}(V T(M) \cap 0)$, we get 


$$
\begin{aligned}
\psi(\zeta)(g w) & =\theta\left(\operatorname{Exp}_{\xi}(\zeta(g w))\right)=\theta\left(\operatorname{Exp}_{\xi}(\operatorname{Tg}(\zeta(w)))\right) \\
& =\theta\left(g \operatorname{Exp}_{\xi}(\zeta(w))\right)=g\left[\theta\left(\operatorname{Exp}_{\xi}(\zeta(w))\right)\right] g^{-1}=g[\psi(\zeta)(w)] g^{-1}
\end{aligned}
$$

Thus we have a $C^{\infty}$-map $\psi: \Gamma_{G}^{r}(V T(M) \cap 0) \rightarrow E$.

We show finally that $\psi \circ \alpha^{*}=\Phi^{-1}$ in a neighborhood of the identity. Choose an open set $V \subset E^{r}(G, M)$ such that $\alpha^{*}(V) \subset \Gamma_{G}^{r}(V T(M) \cap 0)$. Let $h \in V$. Let $f=\Phi^{-1}(h)$. Then

$$
\psi \circ \alpha^{*}(h)=\psi \circ \alpha^{*}(\Phi(f))=\theta \circ \operatorname{Exp}_{\xi} \circ\left(\alpha^{*} \Phi(f)\right) .
$$

So

$$
\begin{aligned}
\left(\psi \circ \alpha^{*}(h)\right)(w) \theta\left(\operatorname{Exp}_{\xi}\left(\left[\alpha^{*} \Phi(f)\right](w)\right)\right) & =\theta\left(\operatorname{Exp}_{\xi}\left(\operatorname{Exp}_{\xi}^{-1}(w, f(w) w)\right)\right) \\
& =\theta(w, f(w) w)=f(w) .
\end{aligned}
$$

Therefore $\psi \circ \alpha^{*}(h)=f=\Phi^{-1}(h)$ as desired. This completes the proof of 4.1.

THeOREM 4.2. $E^{r}(G, M)$ is a Banach Lie group $(r<\infty)$.

Proof. $\Phi$ is a diffeomorphism of the Lie group $E$ onto $E^{r}(G, M)$ and $\Phi$ is an antihomomorphism so the multiplication and inversion in $E^{r}(G, M)$ are smooth.

\section{REFERENCES}

1. R. Abraham, Lectures of Smale on differential topology, Notes at Columbia University, 1962-63.

2. W. D. Curtis, Y.-L. Lee and F. Miller, A class of infinite dimensional subgroups of $\operatorname{Diff}^{r}(X)$ which are Banach Lie groups, Pacific J. Math. 47 (1973), 59-65.

3. H. I. Eliasson, Geometry of manifolds of maps, J. Differential Geometry 1 (1967), 169-194. MR 37 \#2268.

4. S. Kobayashi and K. Nomizu, Foundations of differential geometry. Vol. 1, Interscience, New York, 1963. MR 27 \#2945.

5. J. A. Leslie, On a differential structure for the group of diffeomorphisms, Topology 6 (1967), 263-271. MR 35 \#1041.

6. S. Sternberg, Lectures on differential geometry, Prentice-Hall, Englewood Cliffs, N. J., 1964. MR 33 \#1797.

DEPARTMENT OF MATHEMATICS, KANSAS STATE UNIVERSITY, MANHATTAN, KANSAS 66506 